\title{
Perfil de adolescentes gestantes e de seus recém-nascidos em município do sul do Brasil
}

\author{
Profile of pregnant adolescents and their \\ newborns in a southern Brazilian municipality
}

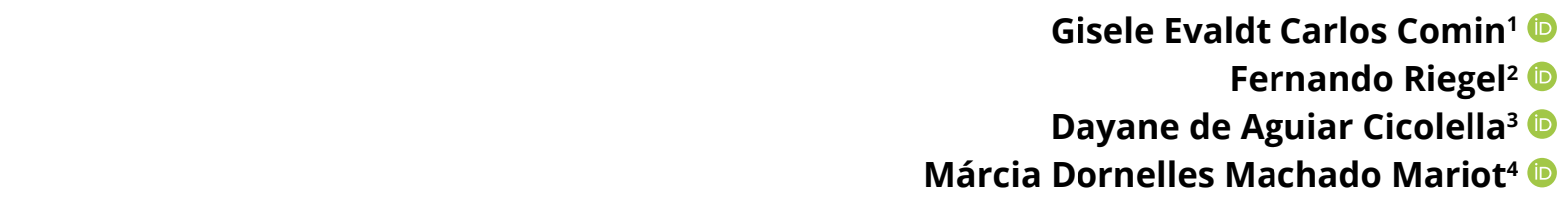

1,3,4Cesuca Centro Universitário (Cachoeirinha). Rio Grande do Sul, Brasil. giselevaldt@gmail.com, dayane.cicolella@cesuca.edu.br, marciamariot@cesuca.edu.br ${ }^{2}$ Autor para correspondência. Universidade Federal de Mato Grosso (Barra do Garças). Mato Grosso, Brasil. fernandoriegel85@gmail.com

RESUMO | OBJETIVO: descrever o perfil de adolescentes gestantes e de seus recém-nascidos em um município do sul do Brasil. MÉTODO: trata-se de um estudo transversal descritivo. A coleta de dados foi realizada com base nos dados das declarações de nascidos vivos do município entre os meses de janeiro a dezembro de 2018. Para avaliar a associação entre as variáveis numéricas, os testes da correlação linear de Pearson ou Spearman foram utilizados. O nível de significância adotado foi de $5 \%(p<0,05)$, utilizando-se medidas de tendência central de variabilidade, frequências absoluta e relativa. RESULTADOS: de 2.974 registros de nascimento, 445 eram de mães adolescentes, resultando num quantitativo de (15\%) de gravidez na adolescência. A maioria das adolescentes possuía idade média de 17,5 anos, (62,5\%) eram brancas, (92,8\%) eram solteiras. Quanto à escolaridade, $61,6 \%$ com o ensino fundamental completo; e (87\%) ocupação do lar. Observouse associação estatisticamente significativa ntre o número de consultas de pré-natal e o peso ao nascer dos neonatos de maneira positiva para a idade das gestantes e contexto de vida. CONCLUSÕES: evidenciou-se um expressivo número de adolescentes gestantes, observando-se a necessidade da implementação de estratégias de planejamento familiar pelos programas de prevenção da gravidez na adolescência.

DESCRITORES: Saúde da Mulher. Gravidez na adolescência. Recém-nascido.

\begin{abstract}
OBJECTIVE: to describe the profile of pregnant adolescents and their newborns in a municipality in southern Brazil. METHOD: this is a cross-sectional descriptive study. Data collection was performed on the basis of data from declarations of live births in the municipality between the months of January to December 2018. The data were analyzed with the aid of the SPSS version 21.0 program. To assess the association between numerical variables, Pearson or Spearman linear correlation tests were used. The level of significance adopted was $5 \%(p<0.05)$ using measures of central tendency of variability, absolute and relative frequencies. The results presented through graphs and tables. RESULTS: out of 2,974 birth records, 445 were from teenage mothers, resulting in a quantity of (15\%) teenage pregnancies. Most adolescents had an average observed age of 17.5 years, (62.5\%) were white, (92.8\%) were single. As for education, $61.6 \%$ had completed elementary school and (87\%) occupied the home. CONCLUSIONS: an expressive number of pregnant adolescents was evidenced, observing the need to implement family planning strategies for adolescent pregnancy prevention programs. The study also made it possible to identify the statistically significant association between the number of prenatal consultations and the birth weight of neonates in a positive way for the age of the pregnant women and the context of life.
\end{abstract}

DESCRIPTORS: Women's health. Teenage pregnancy. Newborn. 


\section{Introdução}

De acordo com o Estatuto da Criança e do Adolescente, considera-se criança o indivíduo até 12 anos e a adolescência é compreendida entre 12 e 18 anos de idade ${ }^{1}$. Nesse período da vida, há mudanças biológicas e psicossociais que conduzem o indivíduo da infância à fase adulta a gravidez nesse período representa um fator adicional com o qual a adolescente precisa lidar².

A gestação na adolescência está associada a dois riscos psicológicos: ser mãe e adolescente. Quando a adolescente engravida, ela passa a desenvolver dois papéis, o de filha e o de mãe, então assim ela terá que redefinir sua identidade, levando em conta que, aqui para frente, sua vida estará vinculada à do filho. A sua projeção para futuro estará afetada, planos serão adiados e serão redirecionados à aternidade e adaptar-se ao papel materno, ao mesmo tempo em que se é filha e adolescente, não será uma tarefa fácil .

A sensibilidade feminina, durante o período gestacional, pode vir a aumentar, ficando a mulher mais suscetível a distúrbios emocionais, dentre eles ansiedade e depressão, afetando assim o vínculo materno-fetal ${ }^{4}$.

O período da adolescência já constitui uma fase de instabilidade emocional; correndo uma gestação precoce, a mulher terá que desenvolver habilidades psicológicas e emocionais de difícil enfrentamento. Por ser essa uma gravidez indesejada ou não programada, o vínculo entre a mãe e o bebê pode ser comprometido pela ausência do apoio familiar, estresse e até mesmo sintomas de depressão ${ }^{5}$.

Outro fato atrelado à gestação na adolescência seria a repetição de uma nova gestação, uma vez que as mulheres que iniciam a maternidade nessa fase da vida possuem um maior número de filhos durante toda a sua vida reprodutiva. Na maioria das vezes, as gestações subsequentes também não são desejadas ou programadas ${ }^{6}$. O presente estudo objetivou descrever o perfil de adolescentes gestantes e de seus recém-nascidos em um município do sul do Brasil.
Trata-se de um estudo transversal descritivo. Este estudo foi realizado na Secretaria Municipal de Saúde do município de Alvorada, do serviço de vigilância epidemiológica, a partir dos dados das Declarações de Nascidos Vivos do município, dos meses de janeiro a dezembro de 2018.

A população do estudo foi constituída por gestantes adolescentes que tiveram filhos nascidos vivos no ano de 2018 no município de Alvorada. Foram excluídas da pesquisa as Declarações de Nascidos Vivos (DNV) que estavam incompletas e as adolescentes que não residiam no município. A coleta de dados foi realizada no mês de abril de 2019, no arquivo da secretaria municipal de saúde. A cidade de Alvorada possui 25 bairros, para essa pesquisa selecionaramse 10 bairros para compor a amostra deste estudo.

Foram avaliadas as variáveis que constam nas Declarações de Nascidos Vivos: variáveis sóciodemográficas da adolescente (idade, escolaridade, ocupação, situação conjugal, raça/cor), variáveis de dados obstétricos (número de gestações anteriores, número de partos vaginais, número de cesáreas, número de nascidos vivos, número de perdas fetais/abortos), dados da gestação atual (data da última menstruação, número de consultas de pré-natal, mês de gestação em que iniciou o pré-natal, tipo de gravidez), dados do parto (apresentação, trabalho de parto induzido, tipo de parto, cesárea ocorreu antes do trabalho de parto iniciar, nascimento assistido por), variáveis sobre o parto (local da ocorrência), variáveis sobre o RN (gênero, peso, raça/cor, índice Apgar, detectada alguma anomalia congênita).

As variáveis quantitativas foram descritas por média e desvio padrão ou mediana e amplitude interquartílica. O teste de Jarque-Bera foi utilizado para avaliar normalidade, pois este apresenta bom desempenho neste tipo de avaliação. As variáveis categóricas foram descritas por frequências absolutas e relativas. Para comparar médias, os testes t-student ou Análise de Variância (ANOVA) foram aplicados. Para avaliar a associação entre as variáveis numéricas, os testes da correlação linear de Pearson ou Spearman foram utilizados. O nível de significância adotado foi de $5 \%$ $(p<0,05)$ e as análises foram realizadas no programa SPSS versão 21.0. 
O projeto foi encaminhado ao Comitê de Ética em Pesquisa (CEP) da Faculdade Inedi - CESUCA onde obteve o aval ético e metodológico para sua execução (CAAE 07069418.5.0000.5665).

\section{Resultados}

Do total de 2.974 registros de nascimento, 445 eram de mães adolescentes, resultando em uma prevalência de 15\% (IC 95\%: 13,8\% a 16,4\%) de gravidez na adolescência. Quanto às condições sóciodemográficas, a média de idade foi de 17,5 anos ( $D P=1,4)$. Predominaram adolescentes da raça/cor branca $(62,5 \%)$, com ensino fundamental $(61,6 \%)$, eram solteiras $(92,8 \%)$ e possuíam a ocupação do lar (87\%). Com relação s situação conjugal observouse que $92,8 \%$ eram solteiras (Tabela 1 ).

Tabela 1. Caracterização sóciodemográfica da amostra das adolescentes. Alvorada, Rio Grande do Sul, Brasil, 2019

\begin{tabular}{|c|c|}
\hline Variáveis & $n=445$ \\
\hline Idade - média \pm DP & $17,5 \pm 1,4$ \\
\hline \multicolumn{2}{|l|}{ Escolaridade - n (\%) } \\
\hline Sem escolaridade & $1(0,2)$ \\
\hline Fundamental $\left(1^{\circ}\right.$ ao $\left.4^{\circ}\right)$ & $9(2,0)$ \\
\hline Fundamental $\left(5^{\circ}\right.$ ao $\left.8^{\circ}\right)$ & $274(61,6)$ \\
\hline Médio & $155(34,8)$ \\
\hline Superior incompleto & $4(0,9)$ \\
\hline Ignorado & $2(0,4)$ \\
\hline \multicolumn{2}{|l|}{ Estado Civil - n (\%) } \\
\hline Solteira & $413(92,8)$ \\
\hline Casada & $20(4,5)$ \\
\hline União estável & $10(2,2)$ \\
\hline Ignorada & $2(0,4)$ \\
\hline \multicolumn{2}{|c|}{ Raça/Cor da Adolescente - n (\%) } \\
\hline Branca & $278(62,5)$ \\
\hline Preta & $67(15,1)$ \\
\hline Parda & $98(22,0)$ \\
\hline Ignorado & $2(0,4)$ \\
\hline \multicolumn{2}{|l|}{ Ocupação - n (\%) } \\
\hline Do lar & $387(87,0)$ \\
\hline Vendedora & $1(0,2)$ \\
\hline Sem ocupação & $1(0,2)$ \\
\hline Estudante & $14(3,1)$ \\
\hline Outras & 42 \\
\hline
\end{tabular}

Fonte: Os autores (2019).

Os dados obstétricos da gestação atual demonstram que a média das semanas de gestação foi de 38,4 semanas, $77,8 \%$ realizaram o parto via vaginal, e $22,2 \%$ por cesárea. A média de consultas de pré-natal foi de 7,2 consultas, sendo que $2,9 \%$ das gestantes não tiveram consultas e 70,6\% fizeram no mínimo 6 consultas. A média do mês da gestação em que as gestantes iniciaram o pré-natal foi de 3,3 meses (Tabela 2). 
Tabela 2. Distribuição das adolescentes segundo dados da gestação atual. Alvorada, Rio Grande do Sul, Brasil, 2019

\begin{tabular}{|c|c|c|}
\hline Variáveis & Media (DP) & n (\%) \\
\hline Semanas de gestação de ocorrência do parto - média \pm DP & & $38,4 \pm 2,3$ \\
\hline Média de consultas pré-natal - média \pm DP & & $7,2 \pm 3,1$ \\
\hline \multicolumn{3}{|l|}{ Número de consultas pré-natal ( $\mathrm{n} / \%$ ) } \\
\hline Mais de 6 & & $314(70,6 \%)$ \\
\hline 1 a 5 & & $118(26,5 \%)$ \\
\hline Nenhuma & & $13(2,9 \%)$ \\
\hline Mês em que iniciou o pré-natal - média \pm DP & & $3,3 \pm 1,5$ \\
\hline \multicolumn{3}{|l|}{ Tipo de gravidez - $\mathrm{n}(\%)$} \\
\hline Única & & $438(98,4 \%)$ \\
\hline Dupla & & $7(1,6 \%)$ \\
\hline \multicolumn{3}{|l|}{ Tipo de parto - $\mathrm{n}(\%)$} \\
\hline Vaginal & & $346(77,8 \%)$ \\
\hline Cesárea & & $99(22,2 \%)$ \\
\hline \multicolumn{3}{|l|}{ Intercorrências com o parto - $n(\%)$} \\
\hline Cesárea ocorreu antes do trabalho de parto & & $34(7,6 \%)$ \\
\hline Parto induzido & & $280(62,9 \%)$ \\
\hline
\end{tabular}

Fonte: Os autores (2019).

Em relação às características dos recém-nascidos, observou-se que $51,7 \%$ eram do sexo masculino e $47,9 \%$, feminino, e 61,7\% eram da raça/cor branca. A média do peso ao nascer foi de $3,14 \mathrm{~kg}$, comprimento de $48,3 \mathrm{~cm}$ e o perímetro cefálico de $33,6 \mathrm{~cm}$. No que se refere ao índice de apgar de primeiro minuto a mediana foi 9; e no quinto minuto também 9. A ocorrência de anomalia congênita foi de 1,1\% (Tabela 3).

Tabela 3. Caracterização dos recém-nascidos. Alvorada, Rio Grande do Sul, Brasil, 2019

\begin{tabular}{|c|c|}
\hline Variáveis & $n=447$ \\
\hline \multicolumn{2}{|l|}{ Sexo-n (\%) } \\
\hline Masculino & $231(51,7)$ \\
\hline Feminino & $214(47,9)$ \\
\hline Ignorado & $2(0,4)$ \\
\hline \multicolumn{2}{|l|}{ Raça/cor - n (\%) } \\
\hline Branca & $276(61,7)$ \\
\hline Preta & $58(13,0)$ \\
\hline Parda & $113(25,3)$ \\
\hline Peso ao nascer (kg) - média \pm DP & $3,14 \pm 0,61$ \\
\hline Comprimento ao nascer $(\mathbf{c m})-$ média $\pm D P$ & $48,3 \pm 3,3$ \\
\hline Perímetro cefálico $(\mathrm{cm})$ - média \pm DP & $33,6 \pm 2,2$ \\
\hline Apgar $1^{\circ}$ minuto - mediana (P25 - P75) & $9(8-9)$ \\
\hline Apgar $5^{\circ}$ minuto - mediana (P25 - P75) & $9(9-10)$ \\
\hline Anomalia Congênita - $n(\%)$ & $5(1,1)$ \\
\hline \multicolumn{2}{|l|}{ Apresentação no parto - $\mathrm{n}(\%)$} \\
\hline Cefálica & $431(96,4)$ \\
\hline Pélvica ou Podálica & $13(2,9)$ \\
\hline Ignorada & $3(0,7)$ \\
\hline
\end{tabular}

Fonte: Os autores (2019). 
A Figura 1, demonstra a associação entre peso ao nascer e número de consultas de pré-natal; houve associação positiva, estatisticamente significativa, entre o número de consultas de pré-natal e o peso ao nascer dos neonatos $(r=0,246 ; p<0,001)$.

Figura 1. Associação entre peso ao nascer e nº de consultas de pré-natal. Alvorada, Rio Grande do Sul, Brasil, 2019

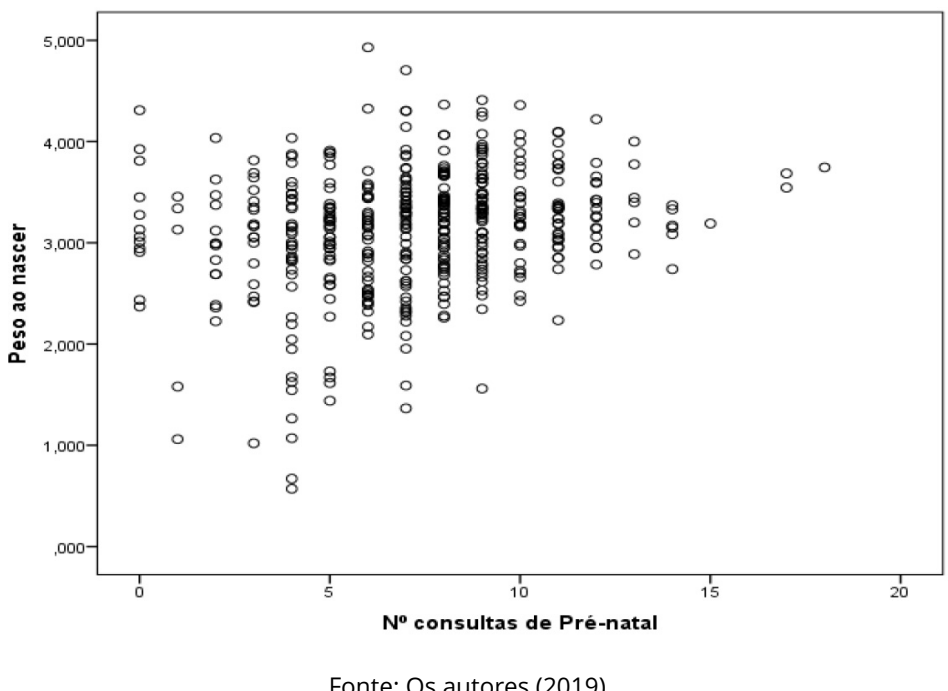

Cabe destacar que não houve associação significativa do número de consultas do pré-natal com a raça/cor da mãe $(p=0,553)$, com o bairro de residência $(p=0,298)$ e nem com a idade da mãe $(p=0,233)$. Havendo, no entanto, uma associação fraca, mas estatisticamente significativa, entre nível de escolaridade e número de consultas do pré-natal $(r s=0,104 ; p=0,029)$. A avaliação do número de consultas de pré-natal e a primiparidade demonstra que as mães que eram primíparas apresentaram maior número de consultas de pré-natal, quando comparadas às não primigestas $(p<0,001)$, conforme demonstra a Figura 2.

Figura 2. Número de consultas de pré-natal e a primiparidade. Alvorada, Rio Grande do Sul, Brasil, 2019

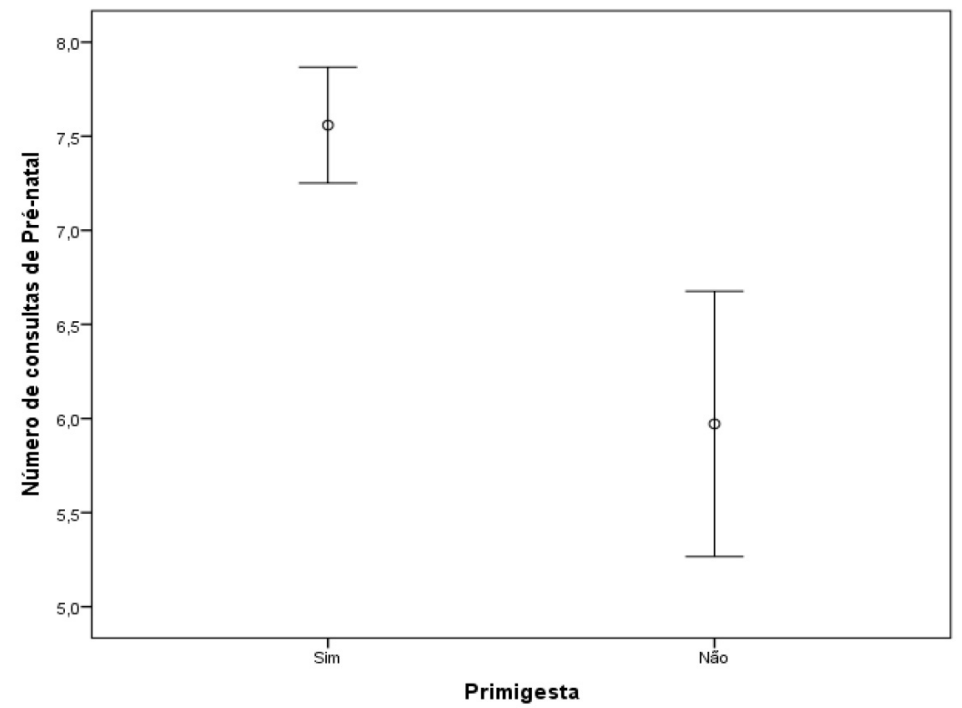

Fonte: Os autores (2019). 


\section{Discussão}

Os principais dados encontrados referentes ao perfil sociodemográfico apontam que a maioria das adolescentes do presente estudo possuía o ensino fundamental dois incompleto, achado semelhante ao do estudo realizado, em 2012, em Londrina, Paraná, com o objetivo de traçar o perfil de gestante adolescente no município, que demonstrou que a maioria das adolescentes $(48,8 \%)$ também possuíam o ensino

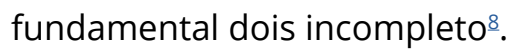

Quanto à prevalência de gestantes adolescentes no município, os dados demostram uma menor prevalência se comparados aos encontrados em uma pesquisa realizado em Vitória da Conquista, Bahia, no ano de 2017, que encontrou uma prevalência de 37\% de gestantes adolescentes no referido município?

Com relação à situação conjugal, a maioria das adolescentes deste estudo eram solteiras, dado este que vai de encontro aos achados do estudo realizado para investigar os fatores a reincidência de gravidez na adolescência, o qual demonstrou que dos $65,5 \%$ de jovens eram casadas $^{10}$.

No tocante à cor da pele da mãe, a maioria eram brancas. Em um estudo realizado para verificar o perfil socioeconômico, demográfico, cultural, regional e comportamental da gravidez na adolescência no Brasil, no ano de 2016, foi observado que a adolescente, por ser branca, reduzia a probabilidade de uma gravidez precoce, fato esse controverso ao resultado obtido ${ }^{11}$.

Quanto à idade média observada, resultados muito semelhantes aos obtidos neste estudo foram descritos em estudo realizado na cidade de Ribeirão Preto, São Paulo, no ano de 2017, para traçar o perfil das gestantes adolescentes usuárias do SUS, no qual a idade média observada foi de 17,3 anos ${ }^{12}$.

No que diz respeito aos dados obstétricos das adolescentes que já estiveram grávidas e aquelas estavam grávidas pela primeira vez, no presente a maioria estava grávida pela primeira vez; os resultados apontam semelhança com o estudo realizado num hospital de acompanhamento de gravidez de alto risco no Ceará, que traçou o perfil das gestantes adolescentes internadas na enfermaria, no qual $82,2 \%$ estavam vivenciando sua primeira gestação, $11,1 \%$ a segunda e $4,44 \%$ a terceira ${ }^{13}$.
Convém ressaltar que a maioria das adolescentes não desenvolvia atividade remunerada, dado semeIhante aos encontrados em estudo realizado na cidade de Piracicaba em São Paulo, no qual 79,4\% das adolescentes não trabalhavam ${ }^{14}$.

O número médio de consultas foi maior do que o preconizado pelo Ministério da Saúde, que prevê um mínimo de 6 consultas, diferentemente dos $40 \%$ das adolescentes avaliadas, em estudo realizado no interior do estado de Minas Gerais em 2017, que tinham menos de seis consultas de pré-natal ${ }^{15}$.

Os resultados do presente estudo referentes à associação positiva estatisticamente significativa, entre o número de consultas de pré-natal e o peso ao nascer dos neonatos, colaboram com os achados obtidos em 2019, em pesquisa realizada no estado do Rio Grande do Norte, que avaliou as condições maternas de adolescentes e o impacto no peso dos neonatos, e que demonstrou haver associação entre o número de consultas de pré-natal e o peso ao nascer ${ }^{16}$.

A média de peso ao nascer do neonato encontrada neste estudo foi semelhante aos resultados observados em estudo realizado no ano de 2017 no município de Diamantina, Minas Gerais, que avaliou os determinantes socioeconômicos e gestacionais do peso ao nascer de crianças nascidas a termo, em que a média de peso ao nascer foi de $3,146 \mathrm{~kg}^{17}$.

O tipo de parto mais prevalente neste presente estudo foi o parto vaginal, dados estes que vão de encontro dos achados aos obtidos em estudo realizado em Goiás para diagnosticar os índices de gravidez e outros fatores relacionados com a gestação na adolescência no ano de 2017, no qual tipo de parto mais prevalente foi o cesáreo ${ }^{18}$.

Os achados desta pesquisa demostram uma elevada prevalência de gestação na adolescência, não sendo muito diferente no restante do país, cabendo ressaltar que se trata de um problema de saúde pública, decorrente do fato de que, cada vez mais, os jovens estão iniciando sua vida sexual precocemente, e os sistemas educacionais e de saúde não têm se mostrado eficientes para mudar essa realidade. Paralelamente a essa situação, a falta de adesão ao pré-natal ou um pré-natal inadequado também são fatores a se destacar, pois causam complicações, tanto para a mãe como para o feto, cabendo lembrar que o pré-natal é um fator de proteção e que, sendo 
realizado adequadamente, promove melhores resultados tanto no parto como no puerpério.

Aponta-se como a principal limitação do presente estudo, O fato da Declaração de Nascido Vivo não possuir informações mais abrangentes, permitindo traçar um perfil social mais completo e as principais condições de vulnerabilidade individuais e programáticas.

\section{Conclusões}

Este estudo possibilitou traçar o perfil sociodemográfico das adolescentes gestantes e de seus recém-nascidos no município de Alvorada, Rio Grande do Sul. Os resultados confirmam que a gestação na adolescência é mais frequente em adolescentes com baixa escolaridade, solteiras e que não desenvolvem atividade remunerada.

Não obstante a gravidez ocorre sem planejamento e em idade precoce, e em relação ao pré-natal, observou uma média adequada de consultas. $O$ peso médio ao nascer dos RN também ficou dentro dos padrões de normalidade e cinco recém-nascidos apresentaram anomalia congênita ao nascer.

O estudo também possibilitou identificar a associação estatisticamente significativa, entre o número de consultas de pré-natal e o adequado peso ao nascer dos neonatos de maneira positiva para a idade das gestantes e contexto de vida.

Por fim, frente à elevada prevalência de gravidez na adolescência, faz-se importante a inclusão dessa temática na agenda dos serviços de saúde, implementando os programas de prevenção da gravidez na adolescência existentes no município pesquisado.

\section{Contribuições dos autores}

Comin GEC participou da concepção, delineamento, busca e análise estatística dos dados da pesquisa, interpretação dos resultados, redação do artigo científico. Cicolella DA participou do delineamento, análise estatística dos dados da pesquisa, interpretação dos resultados. Riegel $F$ participou da análise estatística dos dados da pesquisa, interpretação dos resultados e redação do artigo científico. Mariot MDM participou da concepção, delineamento, análise estatística dos dados da pesquisa, interpretação dos resultados, redação do artigo científico e coordenação do estudo.

\section{Conflitos de interesses}

Nenhum conflito financeiro, legal ou político envolvendo terceiros (governo, empresas e fundações privadas, etc.) foi declarado para nenhum aspecto do trabalho submetido (incluindo, mas não se limitando a subvenções e financiamentos, participação em conselho consultivo, desenho de estudo, preparação de manuscrito, análise estatística, etc.).

\section{Referências}

1. Brasil. Presidência da República. Lei n 8.069, de 13 Julho de 1990. Dispõe sobre o Estatuto da Criança e do Adolescente e dá outras providências. [Internet]. Brasília, DF: 1990. Disponível em: http://www.planalto.gov.br/ccivil_03/leis//8069.htm

2. Rossetto MS, Schermann LB, Beria JU. Maternidade na adolescência: indicadores emocionais negativos e fatores associados em mães de 14 a 16 anos em Porto Alegre, RS, Brasil. Ciênc Saúde Coletiva. 2014;19(10):4235-46. doi: 10.1590/1413$\underline{812320141910.12082013}$

3. Dias ACG, Teixeira MAP. Gravidez na adolescência: um olhar sobre um fenômeno complexo. Paidéia. 2010;20(45):123-31. doi: 10.1590/S0103-863X2010000100015

4. Silva MLFS, Fernandes GAS, Silva JFP, Bezerra EM, Lemos FS, Guedes TG. Gravidez de alto risco: adaptações psicológicas da gestante. Revista Saúde. 2016;10(1).

5. Correia DS, Santos LVA, Calheiros AMN, Vieira MJ. Adolescentes grávidas: sinais, sintomas, intercorrências e presença de estresse. Rev Gaúcha Enferm. 2017;32(1): 40-47. doi: 10.1590/S198314472011000100005

6. Berlofi LM, Alkimin ELC, Barbieri M, Guazzelli C, Araujo FF. Prevenção da reincidência de gravidez em adolescentes: efeitos de um programa de Planejamento Familiar. Acta Paul Enferm. 2006;19(2):196-200. doi: 10.1590/S0103-21002006000200011

7. Polit DF, Beck CT. Fundamentos de pesquisa em enfermagem. 7. ed. Porto Alegre: Artmed; 2011.

8. Pinto KRTF, Bernardy CCF, Morais FR, Gomes K, Cestari MEW, Sodré TM. Gravidez na adolescência: perfil das mães e sua gestação. Uningá Review. 2016;27(2):9-14.

9. Bulhoes TRB, Alves JB, Moreno CA, Silva TB, Dultra LP. Prevalência de recém nascidos pré-termo de mães adolescentes. Revista multidisciplinar e de psicologia. 2017;11(39):84-96. 
10. Nery IS, Gomes KRO, Barros IC, Gomes IS, Fernandes ACN, Viana LMM. Fatores associados à reincidência de gravidez após gestação na adolescência no Piauí, Brasil. Epidemiol Serv Saúde. 2015;24(4):671-80. doi: 10.5123/S1679-49742015000400009

11. Cruz MS, Carvalho FJV, Irffi G. Perfil socioeconômico, demográfico, cultural, regional e comportamental da gravidez na adolescência no Brasil. Planejamento e Políticas Públicas. 2016;46(1):243-66.

12. Vieira EM, Bousquat A, Barros CRS, Alves MCGP. Gravidez na adolescência e a transição para a vida adulta em jovens usuárias do SUS. Rev Saúde Pública. 2017;51(25):1-11. doi: 10.1590/s1518$\underline{8787.2017051006528}$

13. Andrade ACM, Teodósio TBT, Cavalcante AES, Freitas CASL, Vasconcelos MIO, Silva MAM. Perfil das gestantes adolescentes internadas em enfermaria de alto risco em hospital de ensino. SANARE. 2014;13(2):98-102.

14. Fossa AM, Silva TI, Oliveira TS, Rocha MCP, Horibe TM. O perfil de adolescentes grávidas em Piracicaba. Saúde Rev. 2015;15(40):97-109. doi: 10.15600/2238-1244/sr.v15n40p97-109
15. Jezo RFV, Ribeiro IKS, Araujo A, Rodrigues BA. Gravidez na adolescência: perfil das gestantes e mães adolescentes em uma unidade básica de saúde. Revista de enfermagem do centro-oeste mineiro. 2017;7(1):1-8. doi: 10.19175/recom.v7i0.1387

16. Borges EM, Medeiros LNB, Cavalcante AVSON, Melo LGNS. Condição materna de adolescentes e impacto no peso do neonato. Rev Bras Edu Saúde. 2019;9(1):43-49. doi: 10.18378/ rebes.v9i1.6172

17. Moreira MEFH, Silva CL, Freitas RF, Macedo MS, Lessa AC. Determinantes socioeconômicos e gestacionais do peso ao nascer de crianças nascidas a termo. Medicina. 2017;50(2):83-90. doi: 10.11606/issn.2176-7262.v50i2p83-90

18. Pereira LP, Liz M, Assunção PEV. Diagnóstico da gestação na adolescência no Sul de Goiás. Revista da universidade do Rio Verde. 2017;15(2):800-11. doi: 10.18554/reas.v7i3.2884 